\title{
Bow shocks and winds around HD 189733b
}

\author{
J. Llama, A. A. Vidotto, M. Jardine, K. Wood and R. Fares \\ SUPA. University of St Andrews. North Haugh. St Andrews. KY16 9SS. UK \\ email: joe.1lama@st-andrews.ac.uk
}

\begin{abstract}
Asymmetries in exoplanet transits are a useful tool for developing our understanding of magnetic activity on both stars and planets outside our Solar System. Near-UV observations of the WASP - 12 system have revealed asymmetries in the timing of the transit when compared with the optical light curve. In this proceedings we review a number of reported asymmetries and present work simulating near-UV transits for the hot-Jupiter hosting star HD 189733.
\end{abstract}

Keywords. stars: activity, coronae, individual (WASP-12, HD 189733), magnetic fields, planetary systems, winds

\section{Introduction}

An asymmetry has been detected in the HST near-UV light curve of the hot-Jupiter WASP-12b when compared to the optical data. The near-UV light curve shows the transit beginning before the optical transit, but finishing at the same time (Fossati et al. 2010). Vidotto et al. (2010) proposed this early-ingress is caused by the presence of a magnetosphere around the planet. The interaction between the Solar wind and each of the Solar system planets results in the formation of a shock surrounding the planet; however, for magnetised planets, the shock is located further from the planet due to the presence of the planetary magnetosphere. For planets that are located far from their host star, such as Earth and Jupiter, the shock will form directly between the planet and the star, a so-called "dayside shock". For close-in planets, such as hot-Juipiter's, however, an "ahead shock" will form which will occult the stellar disc before the planet which may cause an early-ingress in the transit light curve. If the density of shocked material is high enough then this may show as the presence of additional absorption in the light curve. Llama et al. (2011) modelled the near-UV light curve of WASP-12b from Fossati et al. (2010) and were able to fit the observations with a simple shock model.

In this proceedings we summarize the findings of Llama et al. (2013) where we model a planet and bow shock transiting over a simulated star. We use magnetic maps of the bright K-dwarf HD 189733 (which due to its proximity and relative brightness has been extensively studied) to simulate the stellar wind conditions around the planet transiting planet HD 189733b. From these simulations we are able to prescribe the geometry and density of the shock that should form and simulate near-UV light curves (Llama et al. 2013).

\section{The Model}

We couple numerical simulations of stellar winds with magnetic imaging of HD 189733 to predict the stellar wind conditions around the planet HD 189733b in order to investigate how the geometry of the resultant shock may vary as the planet orbits around the star. We use magnetic surface maps of HD 189733 from June 2007 and also a year later in July 2008 (Fares et al. 2010). The magnetic surface maps are used as one of the boundary conditions in our stellar wind simulation. We use BATS-R-US, a three-dimensional 

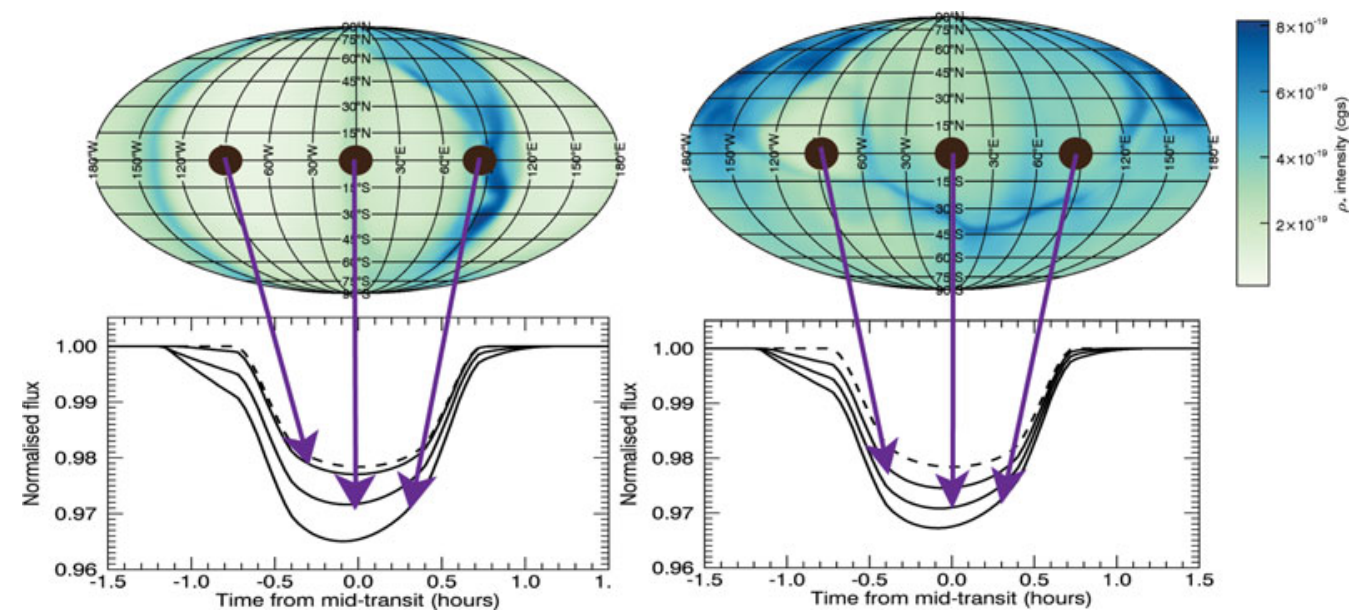

Figure 1. Results of our simulations: June 2007 (left panel) and July 2008 (right panel). Top panel shows the stellar wind density at the orbital distance of the planet. The transit depth and timing is clearly dependent on the local stellar wind conditions for near-UV transits. We find the transits to be very varied, with certain wind conditions causing the transit to be almost indistinguishable from the optical transit, whilst in very dense regions of the wind the transit depth is much deeper and also begins much sooner due to the presence of denser, shocked material.

mangetohydrodynamic (MHD) numerical code developed at The University of Michigan (Powell et al. 1999). The output from the wind simulation is a full three-dimensional grid that allows us to determine the local conditions experienced by the transiting planet HD $189733 \mathrm{~b}$. The formation of a bow shock is a direct consequence of the relative velocities between the interaction of the planetary magnetic field and the stellar wind occurring at supersonic speeds. We use the shock model of Wilkin et al. (1996) coupled with the output of the stellar wind simulations to model the shock around the planet. We are then able to simulate the near-UV light curve as the planet transits over the stellar disc.

\section{Results}

Figure 1 shows our results. The top image shows the stellar wind density at the orbit of HD 189733b for June 2007 (left) and July 2008 (right). The bottom panel shows three resultant near-UV transits (solid lines) and the optical model for comparison (dashed). The results clearly show that the depth and timing of the transit is dependent on the density of the stellar wind and therefore multiple observations may reveal different transit shapes and help us understand the variability of the stellar wind.

\section{References}

Fares, R., Donati, J., Moutou, C., et al. 2010, MNRAS 406, 409

Fossati, L., Haswell, C., Froning, C, et al. 2010, ApJ 714, L222

Llama, J., Wood, K., Jardine, M., Vidotto, A. A. et al., 2011, MNRAS 416, L41

Llama, J., A. A. Vidotto, Jardine, M., Wood, K., Fares, R., Gombosi, T. I. et al., 2011, ArXiv e-prints 1309:2938

Powell, K. G., Roe, P. L., Linde, T. J. et al. 1999, Journal of Computational Physics, 154, 284

Vidotto, A. A., Jardine, M., \& Helling, C. 2010, ApJ, 722, L168 